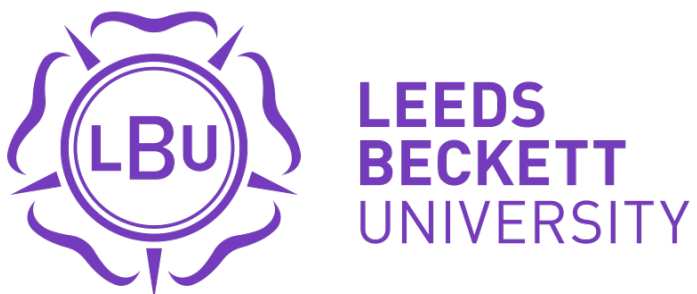

Citation:

Alder, DB and Ford, P and Causer, J and A.Mark, W (2018) The effect of anxiety on anticipation, allocation of attentional resources, and visual search behaviours. Human Movement Science, 61. pp. 81-89. ISSN 0167-9457 DOI: https://doi.org/10.1016/j.humov.2018.07.002

Link to Leeds Beckett Repository record:

https://eprints.leedsbeckett.ac.uk/id/eprint/5132/

Document Version:

Article (Accepted Version)

The aim of the Leeds Beckett Repository is to provide open access to our research, as required by funder policies and permitted by publishers and copyright law.

The Leeds Beckett repository holds a wide range of publications, each of which has been checked for copyright and the relevant embargo period has been applied by the Research Services team.

We operate on a standard take-down policy. If you are the author or publisher of an output and you would like it removed from the repository, please contact us and we will investigate on a case-by-case basis.

Each thesis in the repository has been cleared where necessary by the author for third party copyright. If you would like a thesis to be removed from the repository or believe there is an issue with copyright, please contact us on openaccess@leedsbeckett.ac.uk and we will investigate on a case-by-case basis. 
The effect of anxiety on anticipation, allocation of attentional resources, and visual search behaviours

Alder, D. B.; Ford, Paul; Causer, Joe; A.Mark, Williams 


\begin{abstract}
Successful sports performance requires athletes to be able to mediate any detrimental effects of anxiety whilst being able to complete tasks simultaneously. In this study, we examine how skill level influences the ability to mediate the effects of anxiety on anticipation performance and the capacity to allocate attentional resources to concurrent tasks. We use a counterbalanced, repeated measures design that required expert and novice badminton players to complete a film-based anticipation test in which they predicted serve direction under highand low-anxiety conditions. On selected trials, participants completed an auditory secondary task. Visual search data were recorded and the Mental Readiness Form v-3 was used to measure cognitive anxiety, somatic anxiety and self-confidence. The Rating Scale of Mental Effort was used to measure mental effort. The expert players outperformed their novice counterparts on the anticipation task across both anxiety conditions, with both groups anticipation performance deteriorating under high- compared to low-anxiety. This decrease across anxiety conditions was significantly greater in the novice compared to the expert group. High-anxiety resulted in a shorter final visual fixation duration for both groups when compared to low-anxiety. Anxiety had a negative impact on secondary task performance for the novice, but not the expert group. Our findings suggest that expert athletes more effectively allocated attentional resources during performance under high-anxiety conditions. In contrast, novice athletes used more attentional resources when completing the primary task and, therefore, were unable to maintain secondary task performance under high-anxiety.
\end{abstract}

Key Words: skill acquisition; perceptual-cognitive skill; attention; performance. 


\section{The effects of anxiety on anticipation, allocation of attentional resources and visual search behaviours}

In many professional domains performance can be negatively affected by stressors such as anxiety (e.g., Causer, Holmes, Smith \& Williams, 2011), fatigue (e.g., Casanova, Garganta, Silva, Alves, Oliviera \& Williams, 2013), and injury (e.g., Robbins \& Waked, 1998). Anxiety is defined as "an aversive motivational state that occurs in threatening situations" (Eysenck, Derakshan, Santos, \& Calvo, 2007, p. 336). It can influence various components of performance, including anticipation (Williams \& Elliott, 1999). It is reported that expert athletes reduce the detrimental effects of high-anxiety on performance, possibly by allocating greater attentional resources to the task (Nibbeling, Oudejans, \& Daanen, 2012), reinforcing goal-directed visual search strategies (Wilson, Smith \& Holmes, 2007), and inhibiting feelings of anxiety (Page, Sime, \& Nordell, 1999). However, only a limited number of researchers have investigated the role of expertise in mediating the ability to allocate attentional resources and maintain performance under high-anxiety. We examine this issue using groups of expert and novice badminton players who attempt to anticipate opponent actions when viewing filmed stimuli under high- and low-anxiety conditions.

High-anxiety has been shown to decrease performance in many sports and across expertise levels including the anticipation of karate moves by expert and novice martial artists (Williams \& Elliott, 1999), basketball free throwing by intermediate level players (Wilson, Vine, \& Wood, 2009a), and skeet shooting at the elite level (e.g., Causer, Holmes, Smith, \& Williams, 2011). Several researchers have explored the key skills underpinning high-level performance in badminton (Alder, Ford, Causer, \& Williams, 2014; 2016; Duncan, Chan, Clarke, Cox \& Smith, 2016), with a variety of factors being manipulated including expertise level (skilled $v s$. less-skilled), type of task (serve, smash) and stressors (anxiety, fatigue). The work has consistently highlighted the effects of expertise (Alder et al., 2014), anxiety and 
fatigue (Duncan et al., 2016) on performance, as well as the potential to improve performance through perceptual-cognitive training (Alder, Ford, Williams, \& Causer, 2016).

In Attentional Control Theory (ACT; Eysenck et al., 2007), an explanation is provided as to how anxiety can affect performance. The theory highlights how anxiety can have a negative impact both on performance effectiveness and processing efficiency. Processing efficiency can be measured through changes in underlying mechanisms of performance including mental effort (Wilson et al., 2007) and visual search behaviours (Causer et al., 2011; Williams \& Elliot, 1998; Wilson et al., 2009a; Wilson, Wood \& Vine, 2009b). Performance effectiveness may be calculated by dividing the outcome by the processing resources invested in the task. Under high-anxiety conditions, individuals are thought to allocate attentional resources to locating and negating the source of the threat, which increases mental effort, causing a decrease in performance effectiveness in an effort to maintain performance outcome (Derakshan \& Eysenck, 2009). Vater, Roca, and Williams (2016) describe how when anticipating opponent actions in a temporally occluded 11 vs. 11 soccer test, high-anxiety negatively influenced processing efficiency (as evidenced through increased response times and mental effort) for skilled and less-skilled participants when compared to low-anxiety conditions. However, the effectiveness of performance (i.e., response accuracy) did not change significantly across anxiety conditions.

As well as the proposed reduction in processing efficiency, ACT describes how anxiety alters the contributions of two types of attentional control within working memory, namely the goal-directed and stimulus-driven systems (Baddeley \& Hitch, 1974). The goal-directed system is involved in cognitive control of visual attention and responses, and is influenced by current goals, expectations, and knowledge. The stimulus-driven system is recruited for the detection and direction of attention to relevant, salient or conspicuous events (Corbetta \& Shulman, 2002). Under high-anxiety conditions, ACT proposes that attentional control within working memory 
shifts from the goal-directed system towards the stimulus-driven system. Wilson et al. (2009) presented evidence supporting this shift in attentional control. These authors examined how experienced soccer players executed penalty kicks under high and low-anxiety conditions. In the high-anxiety condition, players fixated for longer durations on the goalkeeper, indicating recruitment of stimulus-driven control, and shorter durations on the target area, demonstrating a decrease in goal-directed focus, when compared to the low-anxiety condition. The decrease in visual attention toward goal-directed sources was accompanied by a decrement in shooting performance.

An integrated model of anxiety and perceptual-motor performance was presented by Nieuwenhuys and Oudejans (2012) to extend and refine on the propositions put forward in ACT. These authors argue that in addition to the threat-related changes in attentional control as a result of high anxiety outlined in ACT, the ability of an individual to correctly interpret information emanating from visual cues is impaired under high-anxiety. They state that although individuals may attend to task-relevant cues (i.e., remaining goal-directed) they may be unable to perceive key information sources correctly. They further argue that the additional effort that accompanies increases in anxiety, as proposed by ACT, can be allocated to a range of tasks involving working memory. First, the additional effort may be directed to reducing the feelings of anxiety. For example, an athlete experiencing anxiety could use pre-determined imagery techniques and breathing strategies to reduce the feelings of anxiety prior to performance (Page et al., 1999). Second, the additional effort may be directed to reinforcing goal-directed attentional strategies or actively inhibiting stimulus-driven processing. For example, researchers have shown that visual search training (e.g., Wilson et al., 2011), in which participants are provided with information relating to the optimal gaze behaviour, can be effective in controlling the impact of anxiety on attentional control. Moreover, placing 
individuals into pressurised situations in training that are congruent to those experienced in competition has been shown to result in improved attentional control (Alder et al., 2016).

The effect of anxiety on performance outcome and processing efficiency may further be related to the expertise level of participants (Nibbeling et al., 2012). It is hypothesised that as expertise level increases, so does the ability to better control the detrimental effects of anxiety on performance (Williams \& Elliott, 1999). It is thought that experts have domain-specific knowledge structures that result in tasks being completed with fewer demands on working memory (Ericsson \& Kintsch, 1995). These lower demands on working memory allow expert athletes to redistribute attentional resources elsewhere, such as when experiencing high-anxiety. In contrast, novices do not have sophisticated domain-specific knowledge structures. Therefore, the high demands of the primary task on working memory do not allow them to redistribute attentional resources under high-anxiety conditions, possibly resulting in decrements to performance outcome when the demands become too great.

In one study, Nibbeling et al. (2012) asked skilled and novice participants to complete a darts throwing task under high- and low-anxiety conditions while carrying out a secondary task of backwards counting. Mental effort and visual search behaviours were measured. In the high-anxiety condition, dart throwing performance was worse for the novice group, but not the skilled group, when compared to the low-anxiety condition. Secondary task performance significantly decreased for both groups in the high- compared to low-anxiety condition. Both groups demonstrated the predicted decrease in processing efficiency, as evidenced by an increase in mental effort and less efficient visual search behaviours, under high- compared to low-anxiety conditions, with this negative change being most pronounced for the less-skilled participants (Eysenck et al., 2007; Nieuwenhuys \& Oudejans, 2012). Cocks, Jackson, Bishop and Williams (2016) reported comparable findings in a study in which skilled and less-skilled tennis players anticipated opponent actions under high- and low-anxiety conditions. The skilled 
players' anticipation performance was superior compared to the less-skilled players, but anxiety did not affect anticipation performance. However, processing efficiency was lower in the highanxiety condition compared to the low-anxiety condition, but skilled players were more efficient than their less-skilled counterparts. Skilled players maintained their superior anticipation performance using less attentional resources when compared to less-skilled players when compensating for the increase in resource demand caused by anxiety, thereby buffering the negative effects of high anxiety on performance effectiveness.

Runswick, Roca, Williams, Bezodis, McRobert, and North (2017) reported contradictory findings in their study involving skilled cricket players. Participants were tasked with playing shots against a live bowler under high- and low-anxiety and under conditions involving the presence of high or low situation-specific context. The high-anxiety manipulation lead to a decrease in performance compared to low-anxiety, with this effect being greater in the high situation-specific context condition compared to the low. These findings suggest participants were not able to delegate attentional resources effectively to produce skilled performance. There is a need to re-examine how skilled participants divide attention under highanxiety conditions to address these contradictory findings.

In the current study, we investigate the ability of expert and novice badminton players to make anticipatory judgements and allocate attentional resources under high- and low-anxiety conditions. Participants completed a temporal occlusion anticipation test under counterbalanced high- and low-anxiety conditions. On selected trials, participants completed a secondary task involving auditory tone monitoring. The expert participants were expected to make more accurate anticipatory judgements compared to their novice counterparts in both anxiety conditions. Both groups were expected to experience a decrease in anticipation judgement accuracy performance outcome in the high- compared to the low-anxiety condition with this decrease was predicted to be more pronounced in the novice group. Processing efficiency was 
predicted to reduce under high-anxiety conditions for both groups compared to the low-anxiety condition, with these effects being more pronounced in novice compared to expert athletes (Nibbeling et al., 2012). We expect a decrease in processing efficiency to be highlighted by an increase in both mental effort, the number of visual fixations employed, a decrease in mean duration of fixation and/or decreased secondary task performance.

\section{Materials and methods}

\section{Participants}

Participants were 10 expert $(M=20$ years of age, $S D=4)$ and 10 novice badminton players $(M=22$ years of age, $S D=2)$. The experts were all professional players and they had accumulated an average of 13 years $(S D=2.4)$ experience in competition. They were engaging in at least 20 hours a week of badminton practice at the time of the study and had played county standard for a minimum of five years in the United Kingdom. The novice participants had not taken part in any structured badminton training or competition. Participants gave their informed consent prior to the study. The local ethics committee provided full ethical approval.

\section{Task and apparatus}

A temporal occlusion test was created involving badminton serves in a doubles match. A total of four expert badminton players of international standard were filmed completing a variety of serves from the first person perspective of their opponent in a doubles match. A highdefinition (HD) video camera (Canon XHA1S; Tokyo, Japan) was positioned two metres away from the net at eye level (1.7 metres). The four players completed three serves to each of six different locations on their opponent's side of the court. The locations were unanimously identified by the panel of three international coaches as being the most commonly used during serves in a badminton doubles match. The six locations were short tee (the point at which the centre line met the service line), short centre, short wide, long tee (the point at which the centre line met the back tramline), long centre, and long wide. During filming, another individual was 
positioned on court to act as the doubles partner for the server. Both the server and their partner could be viewed on the video footage. The film footage was edited (Adobe Premier Pro Editing Software, Version CS5, San Jose, USA) to create video clips to be used as trials in the temporal occlusion test film.

Each video clip or trial began with a black screen for 2,000 ms containing white text informing the participant to stand in the left or right service box so as to receive the on screen serve. At 2,000 ms, a black screen showed white text of a "3, 2, 1" countdown that lasted 3,000 ms. At 5,000 ms, a still picture of the initial video frame of the service action was shown for $1,000 \mathrm{~ms}$. At $6,000 \mathrm{~ms}$, the video clip began playing and the duration of each clip was approximately 3,000 ms. Each clip ended with a black screen that occluded the video and lasted for 3,000 ms. The test film contained 72 trials, involving each of the four servers performing 18 serves comprising three serves to each of the six locations, which were distributed in a random order across the 72 trials. Occlusion points were created to match previous research on anticipation so that clips were occluded $40 \mathrm{~ms}$ prior, $40 \mathrm{~ms}$ after and at shuttle/racket contact (Abernethy, 1990). The three occlusion conditions were each presented 24 times across the 72 trials, and they were equally distributed across trials as a function of the six shot locations.

The test film was back-projected (Epson EB-W05 WXGA 3300 Lumens Projector, Resolution 1280 x 800 pixels, Frequency; $100 \mathrm{~Hz}$ - $120 \mathrm{~Hz}$.) life-size onto a twodimensional screen (size 2.74 metres high x 3.66 metres wide, Draper, USA). The screen was positioned on the opposite side of a full-size international standard badminton court, 1.98 metres from where the net would be, in a position that provided the most representative view of the serves. Participants were required to start each trial on either the left or right hand side of the service area as they would in a normal badminton match. The start locations were marked with an "X" using tape. Participants were required to anticipate the end location of the serve by moving to complete a shadow shot and then verbalising their response. If there was a 
discrepancy between the movement and the verbalised response, the trial was classified as incorrect. The physical shadow return shot was not recorded as a dependent variable, but was used to increase the fidelity of the task. If a participant had not verbalised their answer and completed the shadow return shot by the time the still image for the next trial appeared (i.e. 3,000 ms), the trial was deemed incorrect. No trials were recorded as being inaccurate for the above reasons.

The test sessions were recorded using a high-definition (HD) video camera (Canon XHA1S; Tokyo, Japan) positioned two metres perpendicular to the service line. The video footage was analysed using Dartfish 4.5.2.0 (Dartfish, Fribourg, Switzerland) software with a frequency of $50 \mathrm{~Hz}$ providing an accuracy of $25 \mathrm{~ms} /$ frame. The first movement made by the participants was used as the dependent variable. This was identified as the first frame when there was an "observable and significant lateral motion - right or left - of the racket, the hips, the shoulder or the feet, which was made in order to move to the future location of the next strike" (Triolet et al., 2013, p.822). A correct response corresponded to an initial movement in the same direction as the shuttle direction, while an incorrect response referred to a movement in the opposite direction to where the shuttle was directed. The experimenter hand notated the verbal responses during the experiment.

A secondary task was added to the test film, which consisted of high $(n=18)$ and low $(n=18)$ frequency tones, therefore $50 \%$ of trials $(n=36$ trials $)$ featured a tone. High tones were 2,500 Hz, whereas low tones were $300 \mathrm{~Hz}$. These trials were counter-balanced across occlusion condition, such that each occlusion condition contained six high and six low tones. The tones were presented in such a way that their onset could not be predicted. The tones played between 500 and $700 \mathrm{~ms}$ into the video clip and were presented in a random order, which was kept the same for each participant. Catch trials were used in which either a low tone $(n=18$ trials) or no tone ( $n=36$ trials $)$ were presented in order to make the secondary-task 
unpredictable. Participants held a badminton racket through the experiment, with a push-tomake switch attached to the handle to fit a traditional grip. On high tone trials, participants were instructed to press the button as quickly as possible, whereas on low tone trials they were instructed not to respond. The button was connected to a desktop computer through a cable and synchronised with a developed algorithm through the numerical computing environment MATLAB (Mathworks R2007, UK). The algorithm enabled the onset of the high tones and the moment the participant pressed the button to be recorded and analysed, providing a measure of reaction time for the secondary task.

\section{Procedure}

The experiment consisted of participants completing the primary anticipation task (temporal occlusion test) concurrently with the secondary task on a full-sized international standard badminton court. It involved high- and low-anxiety testing conditions, the order of which was counterbalanced across participants. In total, there were 72 clips or trials of the temporal occlusion test per anxiety condition. In order to limit the potential for learning effects, the trials were randomised in order to create two different test films, which were counterbalanced across participants and anxiety conditions. Prior to the experiment, participants received instruction about the rationale and protocol of the study. They took part in 10 familiarisation trials of the temporal occlusion test prior to starting the experiment.

The level of anxiety experienced by participants during the sessions was manipulated across two separate test sessions using a previously developed protocol (Wilson et al., 2008). In the low-anxiety session, a neutral statement was read to the participants at the start of the session informing them that their performance was to be used for research purposes only and that there would be no consequences for poor performance or comparison to peers. In the highanxiety session, participants were read an anxiety inducing statement at the start of the session in which they were instructed that their performance was being filmed and analysed. The skilled 
group were informed feedback would be provided to their coach and that their performance was to be ranked against their peers, whereas the novice group were instructed they were to be ranked against individuals of similar skill-level and results shown on a notice board. Once the familiarisation trials had finished, regardless of performance, participants in the high-anxiety condition were informed their performance was unsatisfactory and they were to start the test again. Participants were then presented with and interacted with the test film task.

To measure the manipulation of anxiety, participants completed the Mental Readiness Form, version 3 (MRF-3; Krane, 1994). The MRF-3 is a tool used for measuring state anxiety. It has three bipolar 11-point Likert scales that consist of worried and not worried, tense and not tense and, finally, confident and not confident. The MRF-3 was completed after the familiarisation trials in the low-anxiety condition and after the anxiety inducing statement that followed the familiarisation trials in the high-anxiety condition. At the end of both anxiety conditions, participants completed the Rating Scale of Mental Effort (RSME; Zijlstra, 1993). The RSME is a scale ranging from 0-150 with higher scores indicating greater mental effort.

A mobile eye-tracking system (ASL MobileEye, Bedford, USA) was used to record gaze behaviours. The head-mounted monocular eye-tracking system computes point of gaze within a scene through calculation of the vector between pupil and cornea. The calibration consisted of participants fixating six pre-determined locations on a still image of one of the trials (opponent's head and left foot, non-server's head, shuttle, and racket head). During calibration, participants were instructed to adopt the typical stance used when returning serve. The calibration of the eye tracking system was checked after the familiarisation trials.

\section{Data analysis}

Mean scores were calculated from the MRF-3 Likert scales for the two groups in both the high- and low-anxiety condition for each subscale. The data from MRF-3 were analysed via an exploratory 2 Group (Expert, Novice) x 2 Anxiety Condition (High, Low) x 3 subscales 
(Worried, Tense, Confidence) ANOVA. Response accuracy on the primary anticipation task was determined by awarding a correct response for the initial movement that oriented in the same direction as the shuttle landing location, while an incorrect response referred to a movement in the opposite direction to where the shuttle landed. Response accuracy on the primary task was analysed via a 2 Group (Skilled, Novice) x 2 Anxiety Condition (High, Low) ANOVA.

Response time on the secondary task was calculated by determining the difference between the onset of the high tones on each trial and the moment when the button on the racket was pressed. The secondary task analysis was conducted through MATLAB with the software extrapolating all the data points over 4 volts for the button press response. Response time on the secondary task and RSME data were analyzed using separate 2 Group (Expert, Novice) x 2 Anxiety Condition (High, Low) ANOVAs.

The eye movement data were recorded at 25 frames per second with the film footage being subjected to frame-by-frame analysis using video editing software (Adobe Premier Pro Video Editing Software, Version CS 5, San Jose, USA). A fixation was recorded when gaze remained within three degrees of visual angle upon a location for a minimum of $120 \mathrm{~ms}$ (Vickers, 1996). Final fixation was defined as the last fixation on the screen prior to the video occluding. The test film used as the primary task in this study, as well as the eye movement analyses procedures, were the same as in Alder et al. (2014). The servers' action involved two phases. First, a preparation phase starting at the video frame in which the server established their stance by planting their feet $(M=3,400 \mathrm{~ms}, S D=500)$. Second, an execution phase starting from the frame containing the point at which the racket and shuttle are brought together in a "set position" in front of the body up to the frame containing racket-shuttle contact $(M=1,900$ $\mathrm{ms}, S D=500)$. The movement time from the start of the preparation phase to the occlusion point was a mean of $4,300 \mathrm{~ms}$. The analyses of eye movements were conducted from the start 
of the preparation phase of the movement to the occlusion of the video in Alder et al. (2014). Alder et al. reported no between- or within-group differences for fixation location during the preparation phase of the movement, whereas during the execution phase there were expertise and response success main effects and interactions. Given that the duration of the execution phase of the servers' movement is similar to the duration of final fixation, such that the penultimate fixation likely occurs in the preparation phase where no significant differences were found in Alder et al., in the current study only the location of final fixation was analysed.

The number of fixations per trial and mean duration of fixations was calculated. Separate 2 Group (Expert, Novice) x 2 Anxiety Condition (High, Low) ANOVAs were used to analyse the number of fixations per trial, mean duration of fixation, and the mean duration of final fixation. Final fixation location categories were chosen to match those from Alder et al. (2014): racket; wrist; shuttle; head and other. To examine the effect of anxiety and expertise on the final fixation location, an exploratory 2 Group (Expert, Novice) x 2 Anxiety Condition (Low, High) x 5 Location (Racket, Wrist, Shuttle, Head, Other) ANOVA was used with location of fixation being the dependent variable. Intra-reliability observer checks were conducted on the visual search data using the test-retest method (Thomas, Nelson, \& Silverman, 2005), with data from one skilled (97\% reliable) and one novice participant (96\% reliable) being re-analysed.

Tests of normality using Shapiro-Wilk statistics indicated that parametric analyses were appropriate. Any Expertise x Anxiety condition interactions were analysed through computing difference scores (low-anxiety - high-anxiety) for both groups. These scores were then compared using independent samples t-tests. Any other significant interactions were analysed using Tukey's Honestly Significant Difference, whereas Bonferroni comparisons were used for main effects involving more than two variables. Partial eta squared $\left(\eta_{p}{ }^{2}\right)$ was used to represent effect sizes and confidence intervals are presented. The alpha level for significance was adjusted following recommendations presented in Cramer et al. (2016) by controlling familywise error 
rate through the sequential Bonferroni Procedure. That is- $P$ values are presented in ascending order; Alpha values are then adjusted based upon the number of tests run.

\section{Results}

\section{Anxiety manipulation}

The descriptive statistics for the responses to the MRF-3 for both groups across anxiety conditions are presented in Table 1. ANOVA revealed significant main effects of Anxiety Condition, $F(1,18)=44.61, p<.01, \eta_{p}{ }^{2}=.71$, with participants reporting higher anxiety values in the high- compared to the low-anxiety condition. There was no main effect of Group, $F$ (1, $18)=1.76, p=.21, \eta_{p}{ }^{2}=.09$. All interactions were not significant; Subscale $\mathrm{x}$ Group, $F(2,36)$ $=4.65, \mathrm{p}=.018^{1}, \eta_{p}{ }^{2}=.21$, Anxiety Condition x Group, $F(1,18)=.01, \mathrm{p}=.92, \eta_{p}{ }^{2}<.01$, Anxiety Condition x Subscale, $F(2,36)=.79, \mathrm{p}=.46, \eta_{p}{ }^{2}=.04$, or Anxiety Condition x Group x Subscale, $F(2,36)=.22, \mathrm{p}=.81, \eta_{p}^{2}=.01$.

\section{Mental effort}

ANOVA revealed the main effect for anxiety was not significant, $F(1,18)=3.18, p$ $=.09, \eta_{p}^{2}=.15$, there was no group main effect, $F(1,18)=<.01, p=.97, \eta_{p}{ }^{2}<.01$, or Group x Anxiety interaction, $F(1,18)=0.19, p=.66, \eta_{p}^{2}=.01$.

\section{Primary task anticipation performance}

The mean scores for response accuracy for both groups on the anticipation test across the high- and low-anxiety conditions are presented in Figure 1. The mean scores for response accuracy for both groups on the anticipation test across the high- and low-anxiety conditions are presented in Figure 1. ANOVA revealed a significant main effect for group, $F(1,18)=$ $41.51, p<.01, \eta_{p}{ }^{2}=.70$. The skilled group responded more accurately $(M=50$ correct trials out of 72 trials, $S D=6)$, when compared to the novice group $(M=33$ correct trials out of 72 trials, $S D=8)$. There was a significant main effect for anxiety condition, $F(1,18)=4.81, p$ 
$=.04, \eta_{p}{ }^{2}=.21$. Anticipation performance was significantly more accurate in the low- $(M=$ 43 trials, $S D=10)$ compared to high-anxiety condition $(M=40$ correct trials, $S D=12)$. The Group x Anxiety interaction was not significant, $F(1,18)=0.22, p=.65, \eta_{p}^{2}=.01$.

\section{Secondary task performance}

The response times (ms) for both groups on the secondary task across the two anxiety conditions are presented in Figure 2. There was no main effect for group, $F(1,18)=2.31, p$ $=.02^{2}, \eta_{p}{ }^{2}=.27$. There was no main effect for Anxiety Condition, $F(1,18)=2.31, p=.15, \eta_{p}{ }^{2}$ $=.11$. There was a significant Group x Anxiety Condition interaction, $F(1,18)=6.45, p=.02$, $\eta_{p}{ }^{2}=.27$. An independent t-test on the difference scores (Low-anxiety - High-anxiety) tests showed that the response time of the novice group increased significantly more low- to highanxiety condition $(M=94 \mathrm{~ms}, \mathrm{SD}=47)$ compared to the expert group $(M=23 \mathrm{~ms}, S D=138)$, $\mathrm{t}(18)=2.35, \mathrm{p}=.03$. The novice group had significantly slower response times in the highcompared to low-anxiety condition, whereas there was no significant difference in response time between anxiety conditions for the expert group.

\section{Visual search behaviour}

ANOVA revealed no significant main effects or interactions for number of fixations or the mean duration of fixation (for descriptive statistics, see Table 2). For the mean duration of final fixation, there was a group main effect, $F(1,18)=49.34, p<.01, \eta_{p}{ }^{2}=.73$. The final fixation for the expert group was significantly longer compared to the novice group $(M=1,187$ $\mathrm{ms}, S D=195)$. There was a main effect for anxiety condition, $F(1,18)=23.19, p<.01, \eta_{\mathrm{p}}{ }^{2}$ $=.56$. Final fixation was significantly shorter in the high- compared to the low-anxiety condition. The Group x Anxiety condition interaction was not significant, $F(4,72)=0.36, p$ $=.84, \eta_{p}^{2}=.02$.

${ }^{2}$ Non-significant due to alpha value being adjusted to .017 
For fixation location, there were no main effects for group or anxiety condition. There was a main effect for the location of final fixation, $F(4,72)=516.35, p<.01, \eta_{p}{ }^{2}=.97$. The racket was the location of the final fixation on a significantly greater proportion of trials $(M=$ $49 \%$ of all trials, $S D=7)$, compared to the wrist $(M=29 \%$ of all trials, $S D=6)$, shuttle $(M=$ $10 \%$ of all trials, $S D=3)$, head $(M=7 \%$ of all trials, $S D=4)$, and other location $(M=6 \%$ of all trials, $S D=6$ ). The wrist was the location of final fixation on a significantly greater proportion of trials compared to the shuttle, head, and other location. There was no significant difference between the shuttle, head or other location. The Location x Group interaction was significant, $F(4,72)=13.76, p<.01, \eta_{p}^{2}=.43$. The final fixation for the expert group was on the racket and wrist in a greater proportion of trials compared to the novice group, whereas the final fixation for the novice group was on the head and other category in a greater proportion of trials compared to the expert group. There was no significant difference between groups in the proportion of trials that the final fixation was on the shuttle. The three-way Group x Anxiety $\mathrm{x}$ Location interaction was not significant, $F(4,72)=0.36, p=.84, \eta_{p}{ }^{2}=.02$.

\section{Discussion}

We examined the ability of expert and novice badminton players to make anticipation judgements and allocate attentional resources under high- and low-anxiety conditions. As per previous work (Nibbeling et al., 2012; Wilson et al., 2009a), we expected expert participants to make more accurate anticipation judgements compared to their novice counterparts in both anxiety conditions, thus maintaining performance effectiveness as predicted by ACT (Eysenck et al., 2007). The maintenance of performance effectiveness was predicted to be accompanied by a reduction in processing efficiency across anxiety conditions for both expert and novice participants. This decrease in efficiency was predicted to be evidenced through a reduction in secondary task performance, an increase in the number of fixations, a reduction in mean fixation duration, and an increase in mental effort invested on the task (Wilson et al., 2011). Furthermore, 
this increase in mental effort was predicted to be directed to either reducing the feelings of anxiety, as evidenced through no significant differences on the MRF-3 scale (Krane, 1994), or through reinforcing goal-directed strategies, as evidenced by the absence of differences in visual search behaviour patterns across anxiety conditions (Nieuwenhuys \& Oudejans, 2012).

As predicted, the expert group produced significantly more accurate anticipation judgements on the primary task, when compared to the novice group, supporting previous published reports (e.g., Williams et al., 2002; 2012). Moreover, some of the visual search behaviours differed between groups, supporting previous research (Alder et al., 2014; Williams et al., 2002; Williams \& Elliott, 1999). The expertise main effect for anticipation was underpinned by longer final fixations and fixations on more task-relevant information for expert compared to novice participants. It is likely their greater domain-specific experience allows experts to better locate and recognise characteristics within the current environment leading to superior response selection when compared to novices, who do not have the same volume, depth or variety of experience or knowledge (Causer, Janelle, Vickers \& Williams, 2012). The accuracy of anticipation judgements was reduced in the high- compared to low-anxiety condition for both groups. Our findings support previous published reports showing that performance outcome can deteriorate for both novice (e.g., Nibbeling et al., 2012) and skilled participants (e.g., Causer et al., 2011) under high- compared to low-anxiety conditions.

We predicted that processing efficiency would decrease in the high- compared to lowanxiety condition (Eysenck et al., 2007), with this effect being more pronounced in novice compared to expert participants (Cock et al., 2016; Nibbeling et al., 2012). The reduction in processing efficiency was expected to be evidenced through a range of measures. First, the predicted reduction in processing efficiency was evident in the secondary task performance data. Response times for the novices on the secondary task were slower under high- compared to low-anxiety conditions, implying a significant decrease in processing efficiency. However, the 
secondary task performance did not differ between the high- and low-anxiety conditions for the expert group. It appears the effect of high-anxiety did not require the full attentional resources of experts, leading to effective allocation of spare resources to successful secondary task performance, albeit at the expense of primary task performance. The expert group reported higher levels of anxiety compared to the novice group under high-anxiety conditions, perhaps explaining their lack of efficiency in delegating attentional resources to the primary task. In contrast, the novice group appeared to allocate too many attentional resources to the anxiety threat, leading to a lack of resources being available for primary and secondary task performance, explaining the reduction in performance for both tasks as evidenced through a decrease in response accuracy (primary task) and response time (secondary task) under highcompared to low-anxiety. Our findings contradict those reported by Nibbeling et al. (2012) who found that secondary task performance deteriorated under high- compared to low-anxiety conditions for both novice and skilled participants. In their study, the expertise effect as a function of anxiety condition was found for the primary task, but not the secondary task. The differences in anxiety levels experienced or methodological instructions may explain the contradictory findings across studies.

Second, an increase in mental effort was expected under high- compared to low-anxiety conditions as predicted by ACT. However, only weak support for this prediction was reported ( $p=.09$ ). Our findings provide some support for previous research (e.g., Vater et al., 2016; Wilson et al., 2007) and ACT. The data for the cognitive subscale of the MRF-3 may partially explain this effect, as scores on this subscale were greater under high- compared to low anxiety. Nieuwenhuys and Oudejans (2012) suggest that this additional effort may be redirected to a range of specific areas of working memory in order to attempt to maintain performance, such as reinforcing goal-directed attentional control or to reducing the feelings of anxiety. Our MRF3 data showed that participants experienced greater cognitive and somatic anxiety under high- 
compared to low-anxiety conditions, suggesting that participants were not able to reduce the feelings of anxiety. However, our data for final fixation location supports the prediction that participants were reinforcing goal-directed attentional control. In the high-anxiety condition, we expected that the location of the final fixation would be positioned more frequently on less task-relevant (e.g., the head of the server) or threatening sources, as opposed to goal-directed cues (e.g., the racket) (Wilson et al., 2007). In contrast to our predictions, there were no changes in fixation location for either group across the anxiety conditions, suggesting the additional effort was being utilised to reinforce goal-directed strategies. However, final fixation duration was shorter in high- compared to low-anxiety conditions, so although participants were fixating on the same information between anxiety conditions, the shorter period of time potentially led to errors in anticipation judgements. A possible theoretical explanation for this finding is that under high-anxiety participants may have had problems interpreting the key information emanating from the visual cues (Nieuwenhuys \& Oudejans, 2012). It can be postulated, therefore, that regardless of expertise level, under high-anxiety participants could not always perceive or interpret information correctly, perhaps due to the shorter fixation duration, leading to a decrease in anticipation performance.

The absence of significant differences in the number and duration of fixations between high- and low-anxiety may support the prediction that participants were reinforcing goaldirected attentional control. However, a more practical explanation for the lack of change in these visual search behaviours across anxiety conditions may be the constraints of the task. The badminton serve has a short movement duration and short phases within the movement (Alder et al., 2014). Therefore, the short duration of the task may not have provided sufficient time for the differences in fixations normally found across expertise and anxiety levels to become apparent. A limitation of this study is that the secondary task was auditory, rather than visual as per Murray and Janelle (2003). It may be that visual secondary tasks lead to greater 
distractibility from goal-directed cues to less relevant or threatening sensory stimuli. A further limitation relates to the timing of the anxiety measurement. Information relating to anxiety was assessed pre-task in both conditions, post familiarisation trials in the low-anxiety, and post anxiety inducing statement in the high-anxiety condition. Therefore, any changes in levels of anxiety during performance were not concurrently assessed. Furthermore, although the method used to elicit anxiety has been consistently shown to create high levels of anxiety (Alder et al., 2016; Wilson et al., 2007), this may not be truly reflective of the high anxiety conditions experienced by performers in actual competition. In future, researchers should seek to systematically quantify the amount of worrisome thoughts experienced during performance to show how this interacts with the intensity of anxiety and the subsequent effects on performance. Quantifying the amount of worrisome thoughts, perhaps using verbal reports (Fox, Ericsson, \& Best, 2013), would identify the amount of attentional resources being used on irrelevant compared to goal-directed task.

In summary, anticipation accuracy was lower under high- compared to low-anxiety conditions across both groups, supporting previous research with this effect being more pronounced in the novice group (e.g., Causer et al., 2011). Under high-anxiety conditions, there was a decrease in performance efficiency as predicted in ACT for both groups, as evidenced by a decrease in the duration of final fixation. Our visual search data support previous work (i.e., Nieuwenhuys \& Oudejans, 2012). We speculate that the additional effort invested on the task by both groups was used to maintain a goal-directed strategy, potentially shown by a lack of differences in fixation locations across anxiety conditions. Furthermore, our data suggest that although visual search behaviours were mostly maintained, the ability of the participants to correctly interpret the key information emanating from the most relevant areas was hampered under high- compared to low-anxiety. This later finding supports the predictions of Nieuwenhuys and Oudejans (2012) and may be due to a reduction in final fixation duration 
leading to a decrement in anticipation performance. The decrease in secondary task performance for the novice, but not for the expert participants, suggests that experts required fewer attentional resources to perform the primary task, so that under high-anxiety conditions they were able to allocate attentional resources to the effects of anxiety and maintaining secondary task performance. Our data suggest that anxiety negatively impacts performance and its underpinning mechanisms, regardless of expertise level, although experts have greater attentional resources available to deal with high anxiety and maintain at least some aspects of performance when compared novices. 


\section{References}

Abernethy, B. (1990). Anticipation in squash: Differences in advance cue utilization between expert and novice players. Journal of Sport Sciences, 8, 17-34.

Alder, D. B., Ford, P. R., Williams, A. M., \& Causer, J. (2016). The effects of high-and lowanxiety training on the anticipation judgments of elite performers. Journal of Sport and Exercise Psychology, 38, 93-104.

Alder, D., Ford, P. R., Causer, J., \& Williams, A. M. (2014). The coupling between gaze behavior and opponent kinematics during anticipation of badminton shots. Human Movement Science, 37, 167-179.

Baddeley, A. D., \& Hitch, G. J. (1974). Working memory. The Psychology of Learning and Motivation, 8, 47-89.

Behan, M., \& Wilson, M. (2008). State anxiety and visual attention: The role of the quiet eye period in aiming to a far target. Journal of Sport Sciences, 26, 207-215

Casanova, F., Garganta, J., Silva, G., Alves, A., Oliveira, J., \& Williams, A. M. (2013). Effects of prolonged intermittent exercise on perceptual-cognitive processes. Medicine \& Science in Sports \& Exercise, 45(8), 1610-1617.

Causer, J., Holmes, P. S., Smith, N. C., \& Williams, A. M. (2011) Anxiety, movement kinematics, and visual attention in elite-level performers. Emotion, 11, 595- 602.

Causer, J., Janelle, C. M., Vickers, J. N., \& Williams, A. M. (2012). Perceptual expertise: What can be trained? In: Hodges, N. J., \&Williams, A. M. eds. Skill acquisition in sport: Research, theory and practice. London: Routledge. pp 306-324.

Cocks, A.J., Jackson, R.C., Bishop, D.T. and Williams, A.M. (2016) Anxiety, anticipation, and contextual information: A test of Attentional Control Theory. Cognition and Emotion, 30, 1037-1048. 
Corbetta, M., \& Shulman, G. L. (2002). Control of goal-directed and stimulus-driven attention in the brain. Nature Reviews: Neuroscience, 3, 201-215.

Cramer, A. O., van Ravenzwaaij, D., Matzke, D., Steingroever, H., Wetzels, R., Grasman, R. P., \& Wagenmakers, E. J. (2016). Hidden multiplicity in exploratory multiway ANOVA: Prevalence and remedies. Psychonomic Bulletin \& Review, 23, 640-647.

Derakshan, N., \& Eysenck, M. W. (2009). Anxiety, processing efficiency, and cognitive performance new developments from attentional control theory. European Psychologist, $14,168-176$.

Derakshan, N., Smyth, S., \& Eysenck, M. W. (2009). Effects of state anxiety on performance using a task-switching paradigm: an investigation of attentional control theory. Psychological Bulletin Review, 16, 1112-1117.

Duncan, M. J., Chan, C. K., Clarke, N. D., Cox, M., \& Smith, M. (2016). The effect of badminton-specific exercise on badminton short-serve performance in competition and practice climates. European Journal of Sport Science, 1-8.

Ericsson, K. A., \& Kintsch, W. (1995). Long-term working memory. Psychological Review, $102,211-245$.

Eysenck, M. W., Derakshan, N., Santos, R., \& Calvo, M. G. (2007). Anxiety and cognitive performance: attentional control theory. Emotion, 7, 336-353.

Eysenck, M. W., Payne, S., \& Santos, R. (2006). Anxiety and depression: Past, present, and future events. Cognition \& Emotion, 20, 274-294.

Fox, M. C., Ericsson, K. A., \& Best, R. (2011). Do procedures for verbal reporting of thinking have to be reactive? A meta-analysis and recommendations for best reporting methods. Psychological Bulletin, 137 (2), 316. 
Huys, R., Smeeton, N. J., Hodges, N. J., Beek, P. J., \& Williams, A. M. (2008). On the dynamic information underlying visual anticipation skill. Perception \& Psychophysics, 70, 12171234.

Krane, V. (1994). The Mental Readiness Form as a measure of competitive state anxiety. The Sport Psychologist, 8, 89-202

Maracora, S. M., Staiano, W., \& Manning, V. (2009). Mental fatigue impairs physical performance in humans. Journal of Applied Physiology, 106, 857-864.

Murray, N. P., \& Janelle, C. M. (2003). Anxiety and performance: A visual search examination of the Processing Efficiency Theory. Journal of Sport \& Exercise Psychology, 25, 171187.

Nibbeling, N., Oudejans, R. R. D., \& Daanen, H. A. M. (2012). Effects of anxiety, a cognitive secondary task, and expertise on gaze behaviour and performance in a far aiming task. Psychology of Sport and Exercise, 13, 427-435.

Nieuwenhuys, A., \& Oudejans, R. R. (2010). Effects of anxiety on handgun shooting behaviour of police officers: A pilot study. Anxiety, Stress, \& Coping, 23, 225-233.

Nieuwenhuys, A., \& Oudejans, R. R. (2012). Anxiety and perceptual-motor performance: toward an integrated model of concepts, mechanisms, and processes. Psychological Research, 76, 747-759.

Oudejans, R. R. D., \& Pijpers, J. R. (2010). Training with mild anxiety may prevent choking under higher levels of anxiety. Psychology of Sport and Exercise, 11, 44-50.

Page, S. J., Sime, W., \& Nordell, K. (1999). The effects of imagery on female college swimmers' perceptions of anxiety. The Sport Psychologist, 13, 458-469.

Poliszczuk, T., \& Mosakowsk, M. (2009). Inter-reactions of peripheral perception and ability of time-movement anticipation in high class competitive badminton players. Studies in Physical Culture and Tourism, 16, 259-265. 
Reilly, T., Drust, B., \& Clarke, N. (2008). Muscle fatigue during football match-play. Sports Medicine, 38, 357-367.

Robbins, S., \& Waked, E. (1998). Factors associated with ankle injuries. Sports Medicine, 25, 63-72.

Runswick., O., Roca, A., Bezodis, N.E., Williams, A.M. and North, J.S. (2017) The effects of anxiety and situation-specific context on perceptual-motor skill: A multi-level investigation. Psychological Research.

Savelsbergh, G. J., Williams, A. M., Van der Kamp, J., \& Ward, P. (2002). Visual search, anticipation and expertise in soccer goalkeepers. Journal of Sport Sciences, 20,279-287.

Thomas, J. R., Nelson, J. K., \& Silverman, S. J. (2005). Research methods in physical activity (5th ed.). Champaign, IL: Human Kinetics.

Vater, C., Roca, A. \& Williams, A.M. (2016) The effects of anxiety on anticipation and visual search in dynamic, time-constrained situations. Sport, Exercise, and Performance Psychology, 5, 179-192.

Vickers, J. N. (1996). Visual control when aiming at a far target. Journal of Experimental Psychology, 22, 342-354.

Williams, A. M., \& Elliott, D. (1999). Anxiety, expertise, and visual search strategy in karate. Journal of Sport \& Exercise Psychology, 21, 362-375.

Williams, A. M., Ford, P. R., Eccles, D. W., \& Ward, P. (2011). Perceptual-cognitive expertise in sport and its acquisition: Implications for applied cognitive psychology. Applied Cognitive Psychology, 25, 432-442.

Williams, A. M., Vickers, J. N., Rodrigues, S., \& Hillis, F. (2002) Anxiety and performance in table tennis: a test of Eysenck and Calvo's processing efficiency theory. Journal of Sport and Exercise Psychology, 24, 438-455. 
Williams, A. M., Ward, P., Knowles, J. M., \& Smeeton N. J. (2002). Anticipation skill in a realworld task: measurement, training, and transfer in tennis. Journal Experimental Psychology: Applied, 8, 259-270.

Wilson, M., Smith, N. C., \& Holmes, P. S. (2007). The role of effort in influencing the effect of anxiety on performance: Testing the conflicting predictions of processing efficiency theory and the conscious processing hypothesis. British Journal of Psychology, $\quad 98$, 411-428.

Wilson, M. R., Vine, S. J., \& Wood, G. (2009a). The influence of anxiety on visual attentional control in basketball free throw shooting. Journal of Sport Exercise Psychology, 31, 152-168.

Wilson, M. R., Wood, G., \& Vine, S. J. (2009b). Anxiety, attentional control, and performance impairment in penalty kicks. Journal of Sport Exercise Psychology, 31, 761-775.

Zijlstra, F. R. H. (1993). Efficiency in work behaviour: A design approach for modern tools. Delft, the Netherlands: Delft University Press. 